PROCEEDINGS OF THE

AMERICAN MATHEMATICAL SOCIETY

Volume 128, Number 6 , Pages 1859-1867

S 0002-9939(99)05221-1

Article electronically published on September 30, 1999

\title{
CLASP-PASS MOVE AND VASSILIEV INVARIANTS OF TYPE THREE FOR KNOTS
}

\author{
TATSUYA TSUKAMOTO
}

(Communicated by Ronald A. Fintushel)

\begin{abstract}
Recently it has been proved that if and only if two knots $K_{1}$ and $K_{2}$ have the same value for the Vassiliev invariant of type two, then $K_{1}$ can be deformed into $K_{2}$ by a finite sequence of clasp-pass moves. In this paper, we determine the difference of the values of the Vassiliev invariant of type three between two knots which can be deformed into each other by a clasp-pass move.
\end{abstract}

\section{INTRODUCTION}

In [4, K. Habiro defined the $C_{k}$-move and showed the following theorem giving an answer to the question of when two knots take the same value for all Vassiliev invariants of type $k$, where $k(\geq 2)$ is a fixed non-negative integer. We will study the case of $k=1,2$ in this paper.

Theorem 1.1 (Habiro, [4]). Let $k \geq 1$ be an integer and let $K_{1}$ and $K_{2}$ be two knots. Then, the following conditions are equivalent.

(i) $K_{1}$ can be transformed into $K_{2}$ by a finite sequence of $C_{k+1}$-moves.

(ii) $K_{1}$ and $K_{2}$ have the same value for any Vassiliev invariant of type $k$.

We remark here that condition (i) is equivalent to the following condition.

(i') $K_{1}$ can be transformed into $K_{2}$ by a finite sequence of $X_{k+1}$-moves, where the $X_{k}$-move is the following local move defined by Habiro in [3]:

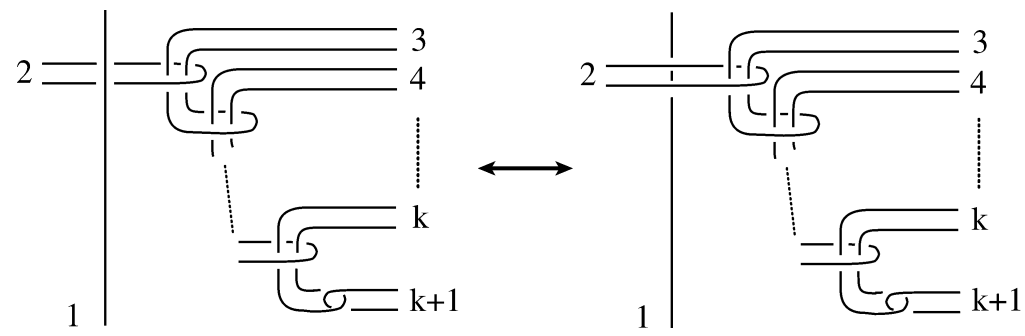

Here we describe terminology used in this paper. For more details we refer the reader to [1], 2]. For a (complex-valued) knot invariant $v_{d}$, we regard it as an

Received by the editors July 31, 1998.

1991 Mathematics Subject Classification. Primary 57M25.

Key words and phrases. Vassiliev invariant, Jones polynomial, clasp-pass move.

(C)2000 American Mathematical Society 
invariant for spatial 4-valent knotted graphs (or singular knots) as follows:

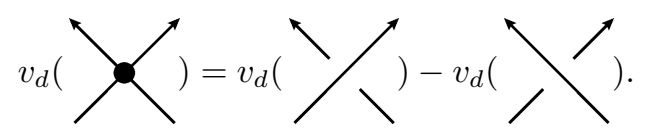

If the graph invariant defined above vanishes for all graphs with more than $d$ vertices, then we call $v_{d}$ a Vassiliev invariant of type $d$. This is equivalent to saying that $v_{d}$ is constant on all graphs with the same $[d]$-configuration. Here a $[d]$-configuration is the cyclically ordered collection of $d$ pairs of $2 d$ points on a circle with dotted lines indicating the pairing. For example,

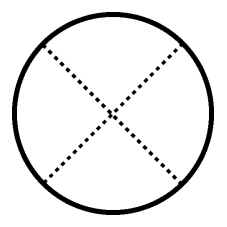

is a [2]-configuration. A singular $\operatorname{knot} G$ with $d$ vertices respects a $[d]$-configuration if each set of paired points forms a vertex in $G$. The following singular knot respects the [2]-configuration above:

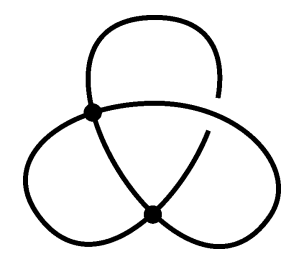

The following equality, called the 4-term relation, holds for any Vassiliev invariant $v$.

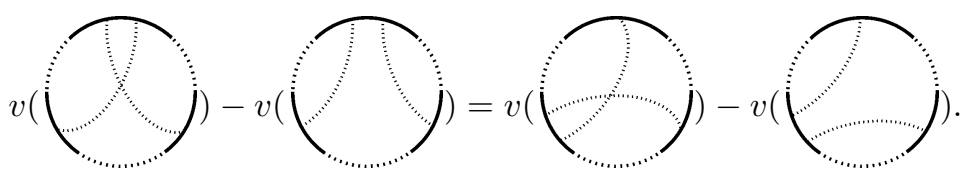

Now we define the local move and the equivalence of local moves.

Definition. Let $K_{1}$ and $K_{2}$ be two knots. Let $t_{1}$ and $t_{2}$ be two 1-manifolds properly embedded in the unit 3-ball $\mathbb{B}^{3}$ with $\partial t_{1}=\partial t_{2}$. We say that we can obtain $K_{2}$ from $K_{1}$ by the local move of type $\left(t_{1} \leftrightarrow t_{2}\right)$ if there is an embedding $h: \mathbb{B}^{3} \rightarrow S^{3}$ such that $h\left(\mathbb{B}^{3}\right) \cap K_{1}=h\left(t_{i}\right)$ and $K_{2} \approx\left(K_{1}-h\left(\mathbb{B}^{3}\right)\right) \cup h\left(t_{j}\right)$, where $(i, j)=(1,2)$ or $(2,1)$ and $\approx$ means an ambient isotopy of $S^{3}$.

Definition. We say that two local moves of type $\left(t_{1} \leftrightarrow t_{2}\right)$ and type $\left(t_{3} \leftrightarrow t_{4}\right)$ are equivalent if there is a homeomorphism $\varphi: \mathbb{B}^{3} \rightarrow \mathbb{B}^{3}$ such that $\varphi\left(t_{i}\right)=t_{3}$ and $\varphi\left(t_{j}\right)=t_{4}$ rel. $\partial \mathbb{B}^{3}$, where $(i, j)=(1,2)$ or $(2,1)$.

We mention here that, for two equivalent local moves of type $\left(t_{1} \leftrightarrow t_{2}\right)$ and type $\left(t_{3} \leftrightarrow t_{4}\right)$, the following conditions are equivalent.

(1) $K_{2}$ is obtained from $K_{1}$ by the local move of type $\left(t_{1} \leftrightarrow t_{2}\right)$.

(2) $K_{2}$ is obtained from $K_{1}$ by the local move of type $\left(t_{3} \leftrightarrow t_{4}\right)$. 


\section{2. $\Delta$-UNKNOTTING OPERATION AND VASSILIEV INVARIANTS OF TYPE TWO}

In this section, we study the $X_{2}$-move and Vassiliev invariants of type two. The $X_{2}$-move is equivalent to the $\Delta$-unknotting operation (Figure 2.1).

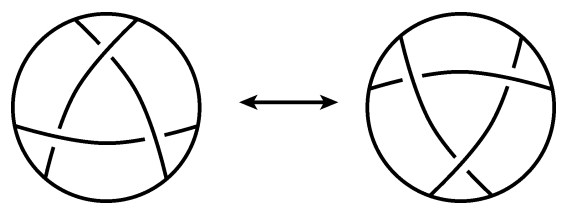

FiguRE 2.1.

The $\Delta$-unknotting operation was defined by H. Murakami and Y. Nakanishi in 8]. They have already proved that every knot can be transformed into a trivial knot by a finite sequence of $\Delta$-unknotting operations, which is a corollary of Theorem 1.1 since the Vassiliev invariant of type one is a constant. The following theorem implies the theorem showed by M. Okada in [9].

Theorem 2.1. Let $K$ and $K^{\prime}$ be two knots that differ by a single $\Delta$-unknotting operation. Then, we have

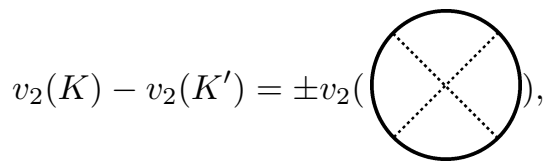

where $v_{2}$ is a Vassiliev invariant of type two.

Corollary 2.2 (Okada, [9]). Let $K$ and $K^{\prime}$ be two knots that differ by a single $\Delta$ unknotting operation. Then, we have $a_{2}(K)-a_{2}\left(K^{\prime}\right)= \pm 1$, where $a_{2}$ is the second coefficient of the Conway polynomial.

Proof of Theorem 2.1. We only show the following since the cases of other orientations can be treated similarly.

$$
v_{2}(K)-v_{2}\left(K^{\prime}\right)=v_{2}\left(\frac{1}{1}\right.
$$


Now we consider the values of their [2]-configurations. Since $K$ is a knot, we may consider two cases as indicated in Figure 2.2 (where dotted lines denote the connecting relations). Thus,

(i)
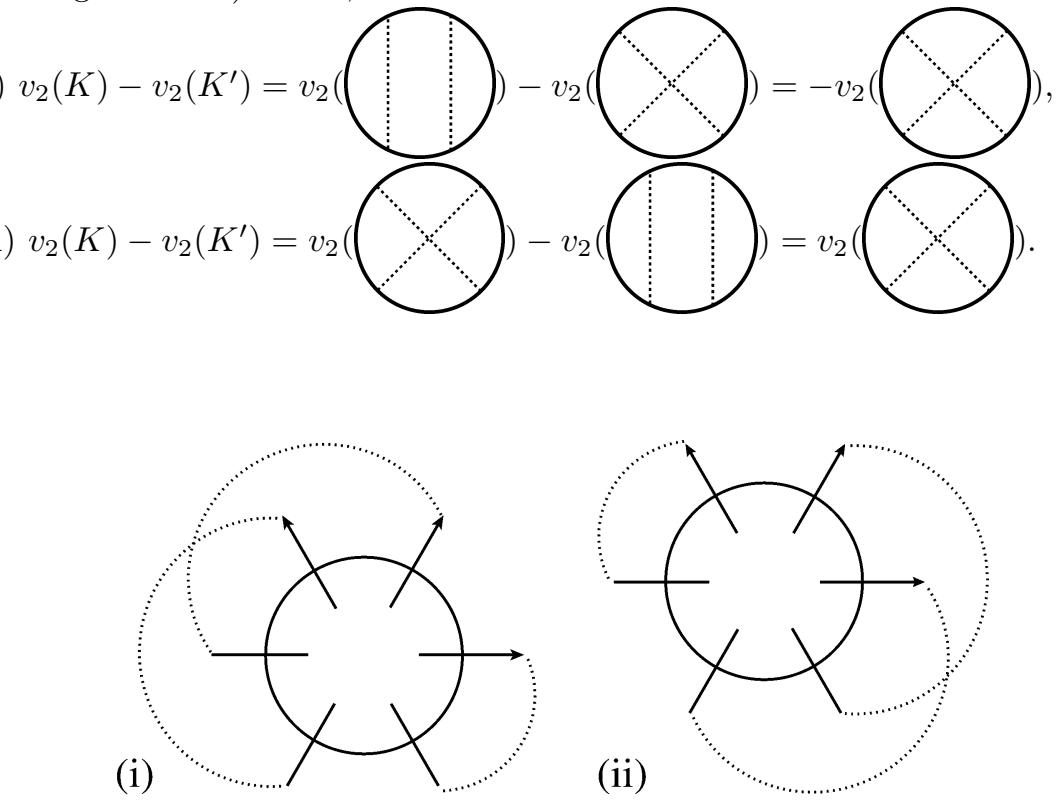

FIGURE 2.2.

\section{Clasp-pass move and Vassiliev invariants of type three}

In this section, we study the $X_{3}$-move and Vassiliev invariants of type three. The $X_{3}$-move is equivalent to the move in Figure 3.1, the clasp-pass move, introduced by Habiro. Figure 3.2 shows the equivalence.

By Theorem 1.1, if $K$ and $K^{\prime}$ have the same value of $a_{2}$, we can define the $C P$ Gordian distance from $K$ to $K^{\prime}$ that is the minimum number of clasp-pass moves which are necessary to deform $K$ into $K^{\prime}$. The following is our main theorem which is useful to determine the distance.

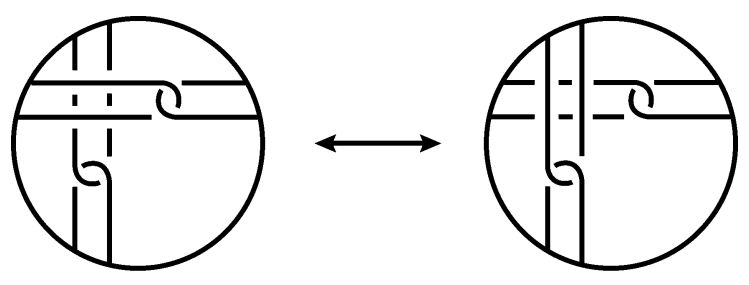

FIGURE 3.1. 


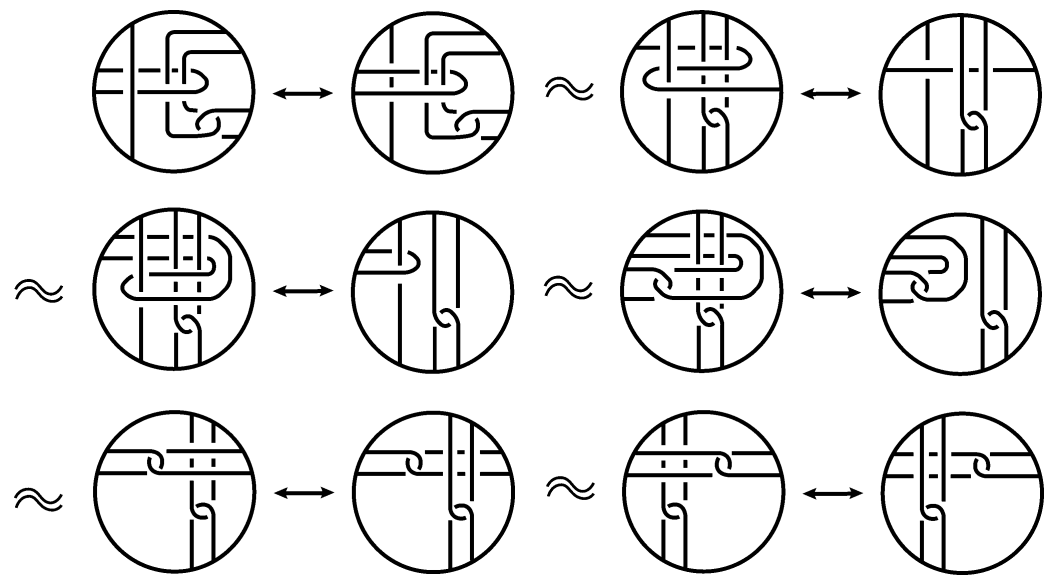

FIGURE 3.2.

Theorem 3.1. Let $K$ and $K^{\prime}$ be two knots that differ by a single clasp-pass move. Then, we have

$$
v_{3}(K)-v_{3}\left(K^{\prime}\right)=0 \quad \text { or } \quad \pm v_{3}(
$$

where $v_{3}$ is a Vassiliev invariant of type three.

Corollary 3.2. Let $K$ and $K^{\prime}$ be two knots that differ by a single clasp-pass move. Then, we have $d^{3} / d t^{3} V_{K}(1)-d^{3} / d t^{3} V_{K^{\prime}}(1)=0$ or \pm 36 , where $V_{K}(t)$ is the Jones polynomial of $K$.

Proof. Here we denote $d^{3} / d t^{3} V_{K}(1)$ simply by $v(K)$. Since $v(K)$ is constant on all singular knots with the same [3]-configuration, we have

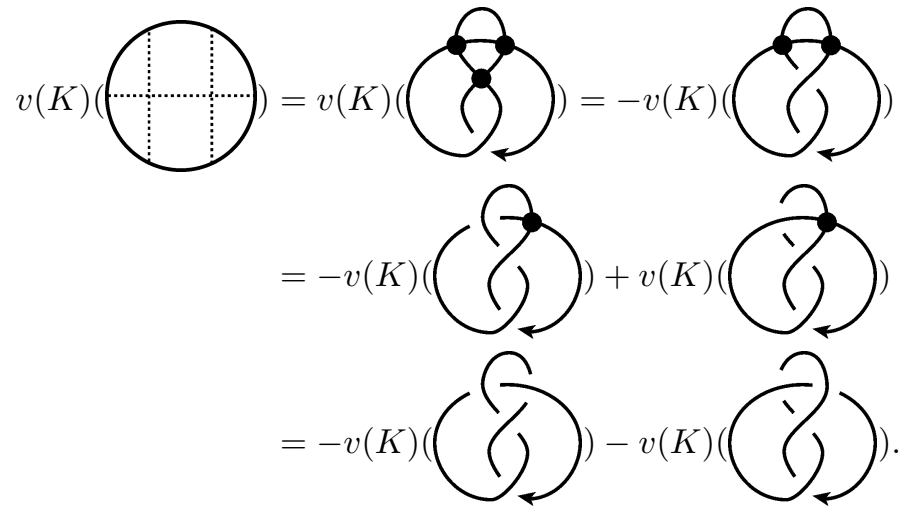
Since $V_{3_{1}}(t)=t+t^{3}-t^{4}$ and $V_{4_{1}}(t)=t^{-2}-t^{-1}+1-t+t^{2}, v(K)$
$18+18=36$. 


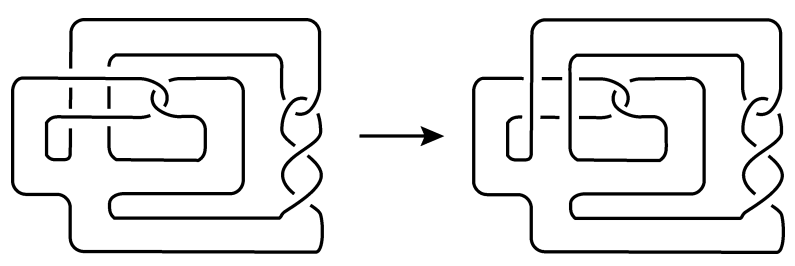

Figure 3.3 .

Remark. Although a single $\Delta$-unknotting operation always changes a knot type, there is a single clasp-pass move which does not change the knot type for any knot. Figure 3.3 shows that there is such a single clasp-pass move for the trivial knot. The case of non-trivial knot follows this example since any knot is a connected sum of itself and the trivial knot.

Proof of Theorem 3.1. We only show the following since the case of the other orientation can be treated similarly.

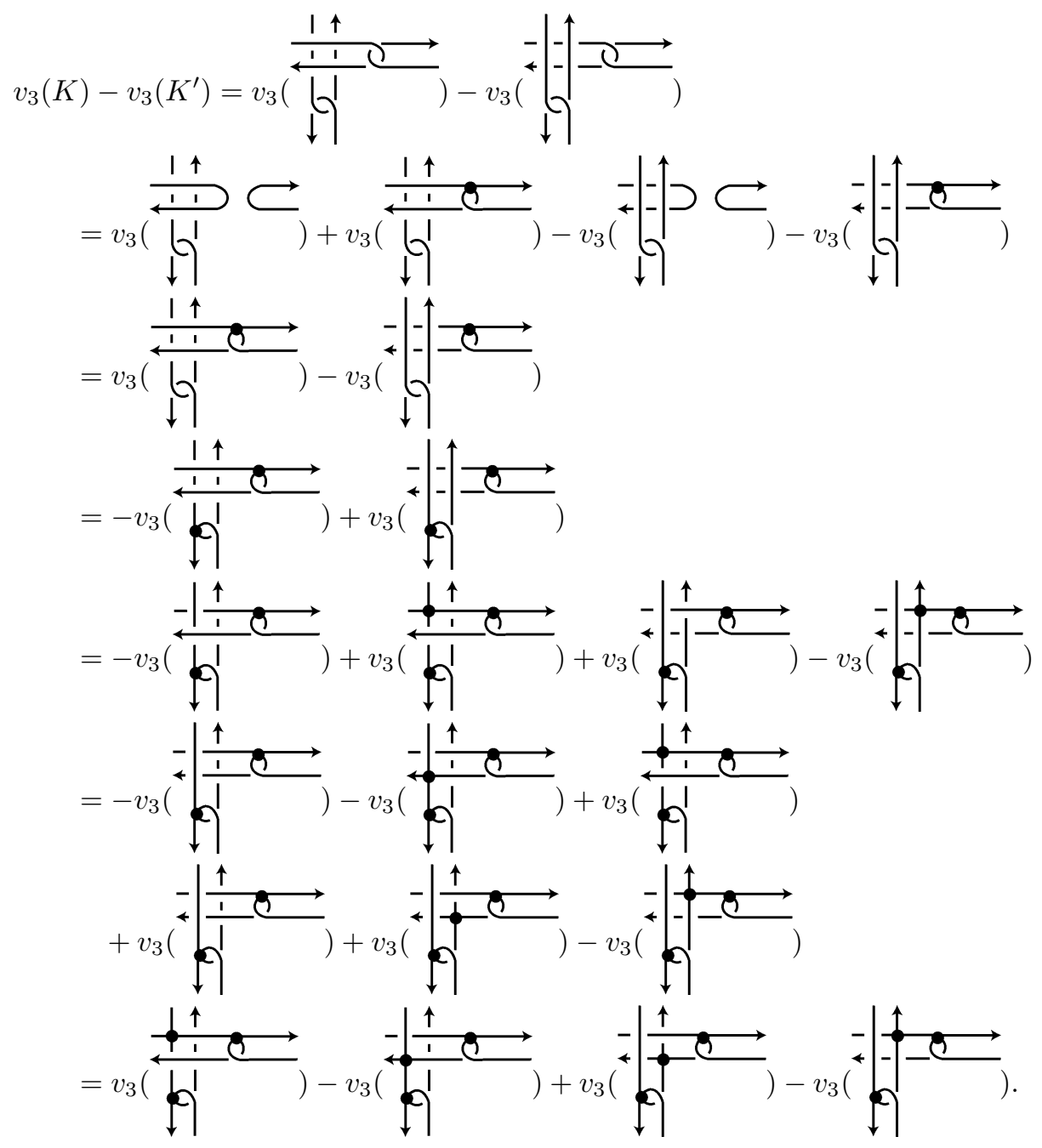


(i)
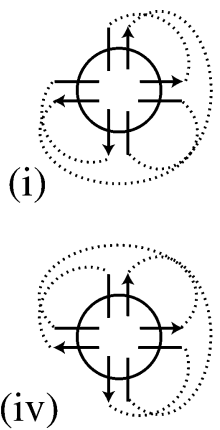

(ii)

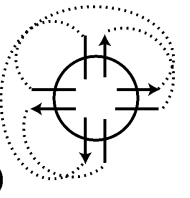

(iii)
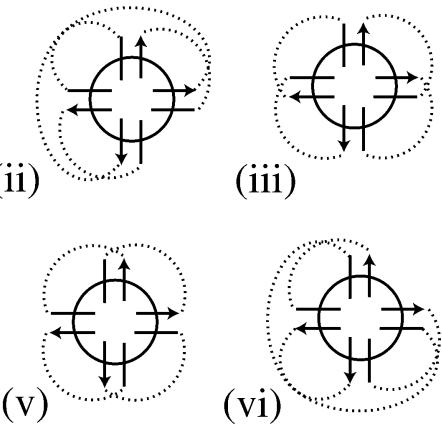

(vi)

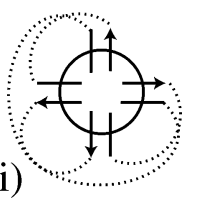

Figure 3.4 .

Now we consider the value of their [3]-configurations. Since $K$ is a knot, we may consider six cases as indicated in Figure 3.4. Thus,

(i)

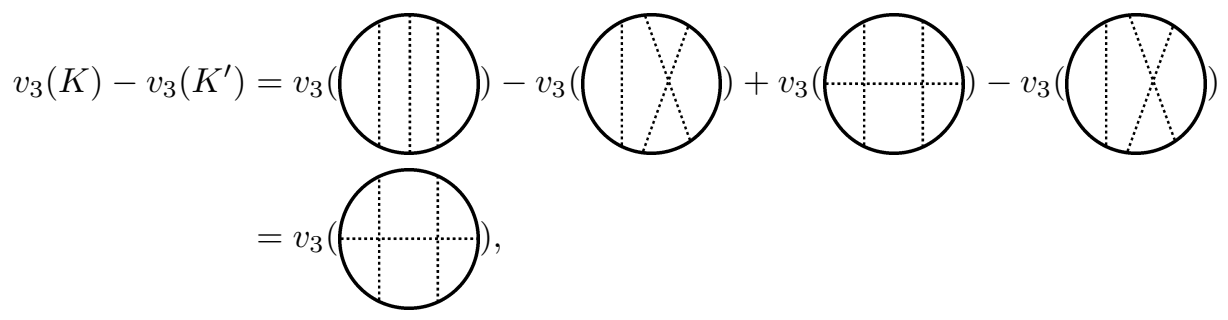

(iii)

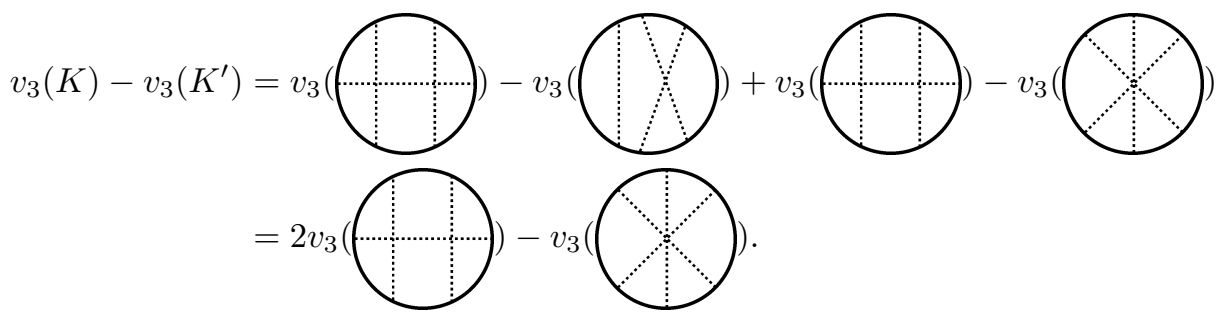

From the 4-term relation, $2 v_{3}\left(\right.$ O. Thus, $v_{3}(K)-v_{3}\left(K^{\prime}\right)=$ 0.

(iv)

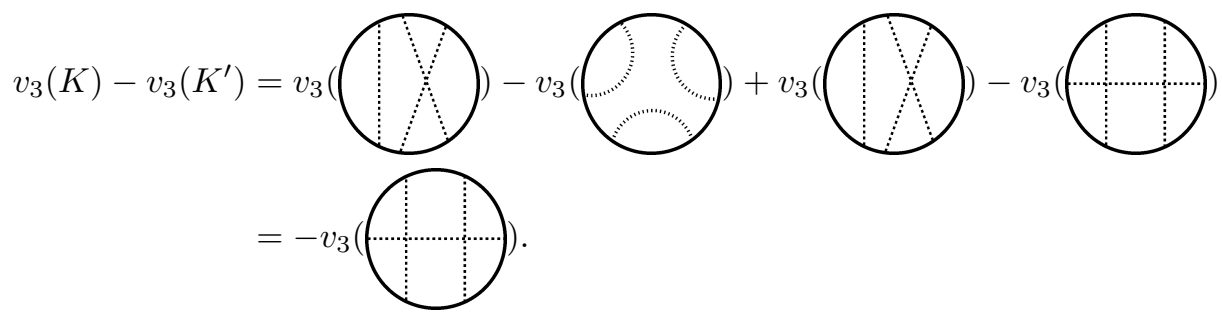

Since the cases (ii), (v) and (vi) can be teated similarly as above, we omit these cases. Thus, we complete the proof. 


\section{Applications}

In this section, we consider knots with $a_{2}$ vanishing.

Example 4.1. Let $K$ be the Kinoshita-Terasaka knot ([6]) and let $0_{1}$ be the trivial knot. Since $a_{2}(K)=0$, we can transform $K$ into $0_{1}$ by a series of clasp-pass moves. In fact, we can accomplish that by carrying out the moves twice (Figure 4.1). Thus, the $C P$-Gordian distance from $K$ to $0_{1}$ is less than or equal to 2 . Since $d^{3} / d t^{3} V_{K}(1)=72$, we can conclude that the distance is exactly equal to 2 from Corollary 3.2.
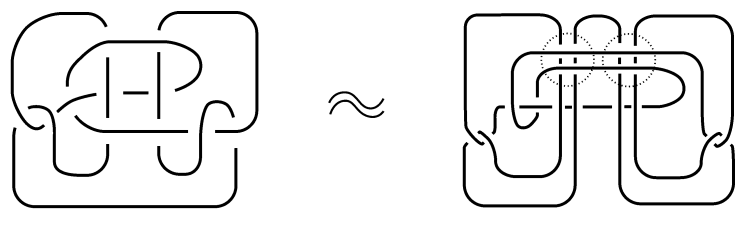

Kinoshita-Terasaka knot

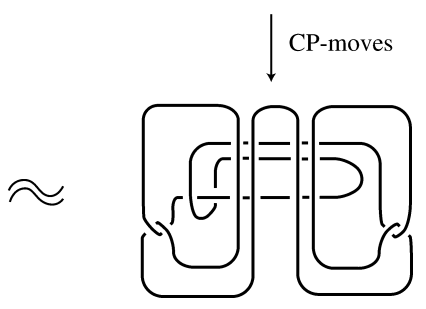

FiguRE 4.1.

Next we show that there are two knots which have the same value of the Vassiliev invariant of type three and can be transformed into each other by a single clasp-pass move.

Example 4.2. Since $a_{2}\left(8_{14}\right)=0$, we can transform $8_{14}$ into $0_{1}$ by a series of clasppass moves. Moreover, $d^{3} / d t^{3} V_{8_{14}}(1)=0$ and we can transform $8_{14}$ into $0_{1}$ by a single clasp-pass move (Figure 4.2).

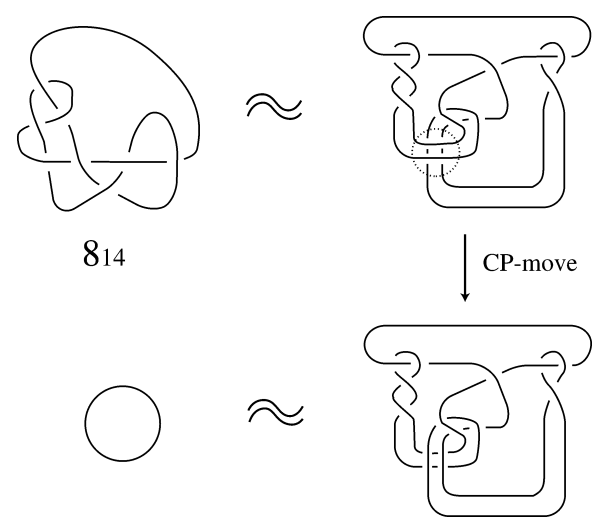

Figure 4.2 . 


\section{ACKNOWLEDGEMENT}

The author is very grateful to Professor Hitoshi Murakami for his helpful comments and encouragements. He would like to thank Dr. Kazuo Habiro for giving valuable advice and an example of a clasp-pass move which does not change the knot type. Thanks are also due to the referee for helpful comments.

\section{REFERENCES}

1. J. S. Birman, New point of view in knot theory, Bull. Amer. Math. Soc. (N.S.) 28 (1993), 253-287. MR 94b:57007

2. J. S. Birman and X. -S. Lin, Knot polynomials and Vassiliev's invariants, Invent. Math. 111 (1993), 225-270. MR 94d:57010

3. K. Habiro, Master thesis of University of Tokyo (1994).

4. K. Habiro, Claspers and the Vassiliev skein modules, preprint, University of Tokyo (1997).

5. K. Habiro, Clasp-pass-moves on knots, preprint, University of Tokyo (1997).

6. S. Kinoshita and H. Terasaka, On unions of knots, Osaka Math. J. 9 (1957), 131-153. MR 20:4846

7. H. Murakami, Some metrics on classical knots, Math. Ann. 270 (1985), 35-45. MR 86g:57007

8. H. Murakami and Y. Nakanishi, On a certain move generating link-homology, Math. Ann. 284 (1989), 75-89. MR 90f:57007

9. M. Okada, Delta-unknotting operation and the second coefficient of the Conway polynomial, J. Math. Soc. Japan 42 (1990), 713-717. MR 91i:57002

Department of Mathematics, School of Science and Engineering, Waseda University, 3-4-1 Okubo, Shinjuku-ku, Tokyo 169-8555, Japan

Department of Mathematics, The George Washington University, Washington, D.C. 20052

E-mail address: tatsuya@gwu.edu 